Proyecciones

Vol. 23, No 1, pp. 1-13, May 2004.

Universidad Católica del Norte

Antofagasta - Chile

\title{
LA FORMA NORMAL DE LIE EN UN CASO CRÍTICO DE UN SISTEMA HAMILTONIANO CON TRES GRADOS DE LIBERTAD Y TRES FRECUENCIAS IGUALES *
}

\author{
JOSÉ MANSILLA \\ Universidad de Magallanes, Chile \\ Received October 2002. Accepted November 2003.
}

\begin{abstract}
In this work, we consider a Hamiltonian system with three degrees of freedom, whose linear part has all its roots pure imaginary and its three frequencies equal. We determine the kernel of the Lie operator and the normal form, according to Meyer of Hamiltonian in the diagonalizable case and in one of the nondiagonalizable cases, obtaining a normal form of the type obtained by Sokol's kii and Mansilla in previous works.
\end{abstract}

AMS classification : Primary 34C20, 34D20, 34K20; Secondary 34D10, 35B34.

Keywords : Sistema Hamiltoniano, resonancias, forma normal.

*Parc. Fin. por Dir. de Investigación UMAG, Proyecto 267-2001. 


\section{Introducción}

La determinación de una forma normal para el Hamiltoniano de un sistema Hamiltoniano, se torna fundamental, sobretodo cuando se quiere estudiar asuntos de estabilidad de soluciones de equilibrio. En este trabajo, y en otros anteriores ([3]), hemos abordado el caso de sistemas Hamiltonianos con tres grados de libertad en los casos críticos, es decir, en los casos en que el sistema linealizado tiene frecuencias iguales. Este tema, lo mismo que el estudio de la estabilidad de las soluciones de equilibrio en tales casos, es no trivial y su estudio ha ofrecido grandes dificultades.

Aquí estudiamos el caso en que el sistema linealizado, del sistema Hamiltoniano, tiene sus tres frecuencias iguales no nulas en los casos diagonalizable y no diagonalizable (uno de los casos) utilizando un método similar al utilizado en [3], presentamos y demostramos los Teoremas 2.1 y 3.1, que dice relación con el núcleo del operador de Lie (parte (I)), y la forma normal del Hamiltoniano respectivo (parte (II)), obteniéndose una forma normal del tipo Sokol'skii ([5]) en el caso del Teorema 3.1.

El planteamiento del problema es el siguiente. Consideremos un sistema Hamiltoniano

$$
\frac{d q_{j}}{d t}=\frac{\partial H}{\partial p_{j}}, \quad \frac{d p_{j}}{d t}=-\frac{\partial H}{\partial q_{j}}, \quad j=1,2,3,
$$

donde el Hamiltoniano $\mathrm{H}$ es dado por

$$
H=H_{0}+H_{1}+H_{2}+\ldots+H_{s}+\ldots
$$

siendo $H_{s}$ un polinomio homogéneo de grado $s+2$ en

$X=\left(q_{1}, q_{2}, q_{3}, p_{1}, p_{2}, p_{3}\right)$. Supongamos que la ecuación caracterí stica del sistema linealizado, determinado por la matriz hamiltoniana $A$, tiene raí ces imaginarias puras del tipo $\pm a i, \pm a i, \quad \pm a i$, con $a$ un número real positivo. Supongamos, además, que la matriz correspondiente está en su forma canónica simpléctica. Entonces la parte cuadrática normalizada, $H^{0}$, es dada por $H^{0}(X)=-\frac{1}{2} X^{T}\left(J A_{3}\right) X$. Por [4], existe un cambio formal simpléctico de coordenadas del tipo, $X=W(Y)=Y+\ldots$ que transforma el Hamiltoniano

$$
H(X)=H_{0}(X)+H_{1}(X)+\ldots
$$

en el Hamiltoniano

$$
H^{*}(Y)=\sum_{s=0}^{\infty} H^{s}(Y)
$$


donde $H_{s}$ y $H^{s}$ son polinomios homogéneos de grado $s+2$ y tal que

$$
H^{s}\left(\varphi^{T}(t, Y)\right) \equiv H^{s}(Y)
$$

para todo $Y \in \mathbb{R}^{6}$ y todo número real $t$, donde $\varphi^{T}(t, Y)$ es el flujo del sistema lineal adjunto determinado por $A^{T}$, es decir,

$$
A^{T}=J R, \quad H_{0}^{T}(X)=\frac{1}{2} X^{T} R X, \text { con } \quad J=\left(\begin{array}{cc}
0 & I_{3} \\
-I_{3} & 0
\end{array}\right) .
$$

Entonces, la condición (1.3) es equivalente a

$$
L_{A^{T}}\left(H^{s}\right)=\left\{H_{0}^{T}, H^{s}\right\}=0, \quad s=1,2,3, \ldots
$$

donde $\{.,$.$\} es el corchete de Poisson ([4]). Este último, L_{A^{T}}$, es llamado operador de Lie y, entonces, cada polinomio homogéneo del Hamiltoniano normalizado se encuentra en su núcleo.

\section{El caso diagonalizable}

Si matriz es diagonalizable, entonces la matriz Hamiltoniana canónica es dada por

$$
A=\left(\begin{array}{cccccc}
0 & 0 & 0 & \delta_{1} a & 0 & 0 \\
0 & 0 & 0 & 0 & a \delta_{2} a & 0 \\
0 & 0 & 0 & 0 & 0 & \delta_{3} a \\
-\delta_{1} a & 0 & 0 & 0 & 0 & 0 \\
0 & -\delta_{2} a & 0 & 0 & 0 & 0 \\
0 & 0 & -\delta_{3} a & 0 & 0 & 0
\end{array}\right)
$$

$\operatorname{con} \delta_{i}= \pm 1, i=1,2,3$. Tenemos el siguiente teorema.

Teorema 2.1. Sea $A$ una matriz hamiltoniana del tipo (2.1). Entonces, en las coordenadas

$$
\zeta_{j}=x_{j}+i y_{j}, \quad \eta_{j}=x_{j}-i y_{j}, \quad(j=1,2,3),
$$

donde $x_{1}, x_{2}, x_{3}, y_{1}, y_{2}, y_{3}$ son coordenadas simplécticas que normalizan un Hamiltoniano con parte lineal determinada por $A$, se tiene, 
I) $\operatorname{Ker}\left(L_{A^{T}}^{s}\right)=\left\{K(\zeta, \bar{\zeta})=\sum_{\substack{(k, l) \in M_{\left(\delta_{1}, \delta_{2}, \delta_{3}\right)}|(k, l)|=s \\ k}} h_{k l} \zeta^{k_{1}} \zeta^{k_{2}} \zeta^{k_{3}} \eta^{l_{1}} \eta^{l_{2}} \eta^{l_{3}}\right.$,

$\left.h_{k l} \in \mathbb{C}\right\}, \quad s>2$, donde

$$
\begin{aligned}
& M_{\left(\delta_{1}, \delta_{2}, \delta_{3}\right)}= \\
& \left\{(k, l)=\left(k_{1}, k_{2}, k_{3}, l_{1}, l_{2}, l_{3}\right) \in \mathbb{Z}_{\geq 0}^{3} \times \mathbb{Z} \geq 0^{3} /\left\langle k-l,\left(\delta_{1}, \delta_{2}, \delta_{3}\right)\right\rangle=0\right\} .
\end{aligned}
$$

II) La forma normal para el Hamiltoniano de un sistema Hamiltoniano con parte lineal determinada por $A$, en las coordenadas

$$
\zeta_{j}=x_{j}+i y_{j}, \quad \eta_{j}=x_{j}-i y_{j}, \quad(j=1,2,3),
$$

tiene la forma

$$
\dot{\zeta}_{j}=\frac{\partial H^{*}}{\partial \eta_{j}}, \quad \dot{\eta}_{j}=-\frac{\partial H^{*}}{\partial \zeta_{j}}, \quad j=1,2,3
$$

donde

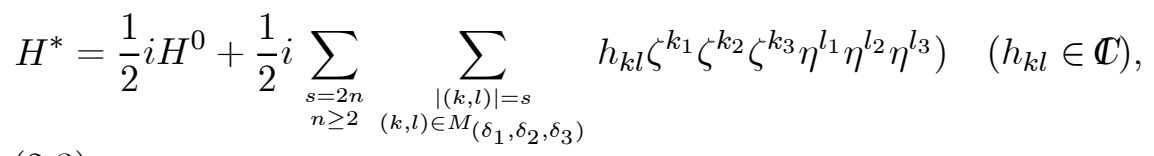

con

$$
H^{0}(x, y)=\frac{\delta_{1}}{2} a\left(x_{1}^{2}+y_{1}^{2}\right)+\frac{\delta_{2}}{2} a\left(x_{2}^{2}+y_{2}^{2}\right)+\frac{\delta_{3}}{2} a\left(x_{1}^{2}+y_{1}^{2}\right)
$$

Demostración : Considerando que $A$ es la matriz hamiltoniana que determina la parte cuadrática de un Hamiltoniano, entonces en coordenadas simplécticas $\left(q_{1}, q_{2}, q_{3}, p_{1}, p_{2}, p_{3}\right)$, éste es dado por

$$
H_{0}=\frac{\delta_{1}}{2} a\left(q_{1}^{2}+p_{1}^{2}\right)+\frac{\delta_{2}}{2} a\left(q_{2}^{2}+p_{2}^{2}\right)+\frac{\delta_{3}}{2} a\left(q_{1}^{2}+p_{1}^{2}\right) .
$$

La matriz transpuesta de $A$ es dada por

$$
R=A^{T}\left(\begin{array}{cccccc}
0 & 0 & 0 & -\delta_{1} a & 0 & 0 \\
0 & 0 & 0 & 0 & -\delta_{2} a & 0 \\
0 & 0 & 0 & 0 & 0 & -\delta_{3} a \\
a \delta_{1} & 0 & 0 & 0 & 0 & 0 \\
0 & a \delta_{2} & 0 & 0 & 0 & 0 \\
0 & 0 & a \delta_{3} & 0 & 0 & 0
\end{array}\right)
$$


$\operatorname{con} \delta_{i}= \pm 1, i=1,2,3 \mathrm{y}$,

entonces, el Hamiltoniano cuadrático para el sistema adjunto es dado por

$$
H_{A^{T}}^{0}=\frac{-\delta_{1}}{2} a\left(q_{1}^{2}+p_{1}^{2}\right)+\frac{-\delta_{2}}{2} a\left(q_{2}^{2}+p_{2}^{2}\right)+\frac{-\delta_{3}}{2} a\left(q_{1}^{2}+p_{1}^{2}\right) .
$$

De esta forma, el operador de Lie $L_{A^{T}}$, para un polinomio $K$ es dado por

$$
\begin{aligned}
L_{A^{T}}(K)=\delta_{1} a & \left(p_{1} \frac{\partial K}{\partial q_{1}}-q_{1} \frac{\partial K}{\partial p_{1}}\right)+\delta_{2} a\left(p_{2} \frac{\partial K}{\partial q_{2}}-q_{2} \frac{\partial K}{\partial p_{2}}\right) \\
+ & \delta_{3} a\left(p_{3} \frac{\partial K}{\partial q_{3}}-q_{3} \frac{\partial K}{\partial p_{3}}\right) .
\end{aligned}
$$

Suponiendo que en las coordenadas $x_{j}, y_{j}$ el Hamiltoniano está normalizado, hagamos el cambio de variable

$$
\zeta_{j}=x_{j}+i y_{j}, \quad \eta_{j}=x_{j}-i y_{j}, \quad(j=1,2,3) .
$$

Observemos que este cambio de variables es simpléctico de multiplicador $-2 i$. Entonces, para un monomio de la forma $K=\zeta^{k_{1}} \zeta^{k_{2}} \zeta^{k_{3}} \eta^{l_{1}} \eta^{l_{2}} \eta^{l_{3}}$ $\left(k_{j}, l_{j} \in Z_{\geq 0}, \mathrm{j}=1,2,3\right)$ obtenemos que

$$
L_{A^{T}}(K)=i a\left[\delta_{1}\left(l_{1}-k_{1}\right)+\delta_{2}\left(l_{2}-k_{2}\right)+\delta_{3}\left(l_{3}-k_{3}\right)\right] K .
$$

Consideremos el subconjunto de $Z^{3} \times Z^{3}$ definido por

$$
\begin{aligned}
& M_{\left(\delta_{1}, \delta_{2}, \delta_{3}\right)}= \\
& \left\{r=(k, l)=\left(k_{1}, k_{2}, k_{3}, l_{1}, l_{2}, l_{3}\right) \in Z_{\geq 0}^{3} \times Z_{\geq 0}^{3} /\left\langle k-l,\left(\delta_{1}, \delta_{2}, \delta_{3}\right)\right\rangle=0\right\}
\end{aligned}
$$

entonces, un polinomio homogéneo de la forma

$K=\sum_{|(k, l)|=s} h_{k l} \zeta^{k_{1}} \zeta^{k_{2}} \zeta^{k_{3}} \eta^{l_{1}} \eta^{l_{2}} \eta^{l_{3}}$, con $s>0,(k, l)=\left(k_{1}, k_{2}, k_{3}, l_{1}, l_{2}, l_{3}\right) \in$ $Z_{\geq 0}^{3} \times Z_{\geq 0}^{3} \mathrm{y}|(k, l)|=k_{1}+k_{2}+k_{3}+l_{1}+l_{2}+l_{3}$, está en $\operatorname{Ker}\left(L_{A^{T}}^{s}\right)$ si y sólo si, todos los coeficientes no nulos $h_{k l}$ de $K$ son tales que $(k, l) \in M_{\left(\delta_{1}, \delta_{2}, \delta_{3}\right)}$, es decir,

$$
\operatorname{Ker}\left(L_{A^{T}}^{s}\right)=\left\{K(\zeta, \bar{\zeta})=\sum_{\substack{|(k, l)|=s \\(k, 1) \in M_{\left(\delta_{1}, \delta_{2}, \delta_{3}\right)}}} h_{k l} \zeta^{k_{1}} \zeta^{k_{2}} \zeta^{k_{3}} \eta^{l_{1}} \eta^{l_{2}} \eta^{l_{3}}, \quad h_{k l} \in C\right\}
$$

Por lo que hemos demostrado la parte (I) del Teorema.

Siguiendo, un Hamiltoniano con parte cuadrática determinada por $A$ de la forma

$$
H^{*}(x, y)=H^{0}+H^{1}+H^{2}+\ldots+H^{s}+\ldots
$$

donde $H^{s}$ es un polinomio de homogéneo de grado $s+2$ en 
$\left(x_{1}, x_{2}, x_{3}, y_{1}, y_{2}, y_{3}\right)$, está normalizado $(2.2)$ si para $s>0, H^{s} \in \operatorname{Ker}\left(L_{A^{T}}\right)$, por lo que en las variables dadas en (7) debe tenerse que

$$
H^{s}\left(\zeta^{k}, \eta^{l}\right)=\sum_{\substack{|(k, l)|=s+2 \\(k, 1) \in M_{\left(\delta_{1}, \delta_{2}, \delta_{3}\right)}}} h_{k l} \zeta^{k_{1}} \zeta^{k_{2}} \zeta^{k_{3}} \eta^{l_{1}} \eta^{l_{2}} \eta^{l_{3}}
$$

Supongamos que $H^{s} \neq 0$, entonces, para un término de sub-índice $(k, l)$ se tiene las relaciones

$$
\begin{gathered}
k_{1}+k_{2}+k_{3}+l_{1}+l_{2}+l_{3}=s+2 \\
\delta_{1} k_{1}+\delta_{2} k_{2}+\delta_{3} k_{3}=\delta_{1} l_{1}+\delta_{2} l_{2}+\delta_{3} l_{3}
\end{gathered}
$$

de donde obtenemos, independientemente de los $\delta$ 's que $s+2$ debe ser un número par, es decir, $s$ es un número par y, entonces, los únicos términos $H^{s}$ no nulos en el Hamiltoniano normalizado son los de grado par. Rescribiendo $H^{*}$ con estas consideraciones, tenemos demostrado la parte (II) del Teorema.

\section{Un caso no diagonalizable}

Estudiamos aquí , el caso en que la matriz del sistema linealizado es no diagonalizable y tiene la forma canónica simpléctica $([1],[2])$

$$
A=\left(\begin{array}{cccccc}
0 & 0 & 0 & \delta_{1} a & 0 & 0 \\
0 & 0 & a & 0 & 0 & 0 \\
0 & -a & 0 & 0 & 0 & 0 \\
-\delta_{1} a & 0 & 0 & 0 & 0 & 0 \\
0 & \delta_{2} & 0 & 0 & 0 & a \\
0 & 0 & \delta_{2} & 0 & -a & 0
\end{array}\right)
$$

con $\delta_{i}= \pm 1, i=1,2$. Entonces la parte cuadrática normalizada, $H^{0}$, de un Hamiltoniano con parte lineal determinada por $A$, es dada por

$$
\begin{aligned}
H_{0}(X) & =-\frac{1}{2} X^{T}\left(J A_{3}\right) X \\
& =\frac{\delta_{1} a}{2}\left(q_{1}^{2}+p_{1}^{2}\right)+\frac{\delta_{2}}{2}\left(q_{2}^{2}+q_{3}^{2}\right)+a\left(q_{3} p_{2}-q_{2} p_{3}\right)
\end{aligned}
$$

donde $X=\left(q_{1}, q_{2}, q_{3}, p_{1}, p_{2}, p_{3}\right)$, son coordenadas simplécticas. 
Considerando que las variables que llevan al Hamiltoniano (1.2) a su forma normal completa son $\left(x_{1}, x_{2}, x_{3}, y_{1}, y_{2}, y_{3}\right)$, tenemos el siguiente teorema.

Teorema 3.1. Sea $A$ una matriz hamiltoniana del tipo (3.1). Entonces, en las coordenadas simplécticas $x_{1}, x_{2}, x_{3}, y_{1}, y_{2}, y_{3}$, se tiene que,

I) Para $s>2$,

$$
\operatorname{Ker}\left(L_{A^{T}}^{s}\right) \subseteq \mathbb{R}\left[\Gamma_{1}, \Gamma_{2}, \Gamma_{3}, \Gamma_{4}\right],
$$

más especí ficamente, si $K^{s} \in \operatorname{Ker}\left(L_{A^{T}}^{s}\right)$ entonces $K^{s}$ es un polinomio homogéneo de la forma

$$
K^{s}=\sum_{\left|\left(n_{1}, n_{2}, n_{3}, n_{4}\right)\right|=s} h_{n} \Gamma_{1}^{n_{1}} \Gamma_{2}^{n_{2}} \Gamma_{3}^{n_{3}} \Gamma_{4}^{n_{4}} \quad\left(h_{n} \in \mathbb{R}\right),
$$

donde

$$
\Gamma_{1}=\frac{1}{2}\left(x_{1}^{2}+y_{1}^{2}\right), \quad \Gamma_{2}=\frac{1}{2}\left(y_{2}^{2}+y_{3}^{2}\right), \quad \Gamma_{3}=x_{3} y_{2}-x_{2} y_{3}, \quad \Gamma_{4}=\frac{1}{2}\left(x_{2}^{2}+x_{3}^{2}\right) .
$$

II) Consideremos un sistema Hamiltoniano del tipo (1.2) con las condiciones de la parte lineal anteriores. Entonces la forma normal , $H^{*}$, para el Hamiltoniano (1.2) expresado en las variables $\left(x_{1}, x_{2}, x_{3}, y_{1}, y_{2}, y_{3}\right)$ tiene la forma,

$$
H^{*}(x, y)=H^{0}+H^{1}+H^{2}+\ldots+H^{s}+\ldots
$$

donde $H^{s}, s \geq 1$, es un polinomio homogéneo de grado $2(s+1)$ en $x_{1}, x_{2}, x_{3}, y_{1}, y_{2}, y_{3}$ y un polinomio homogéneo de grado $s+1$ en $\Gamma_{1}$, $\Gamma_{2}, \Gamma_{3}$ y con $H^{0}=\delta_{1} a \Gamma_{1}+a \Gamma_{3}+\delta_{2} \Gamma_{4}$.

\section{Demostración.}

I) Con las hipótesis anteriores, aquí

$$
R=\left(\begin{array}{cccccc}
-\delta_{1} a & 0 & 0 & \delta_{1} a & 0 & 0 \\
0 & 0 & 0 & 0 & 0 & a \\
0 & 0 & 0 & 0 & -a & 0 \\
0 & 0 & 0 & -\delta_{1} a & 0 & 0 \\
0 & 0 & -a & 0 & -\delta_{2} & 0 \\
0 & a & 0 & 0 & 0 & -\delta_{2}
\end{array}\right)
$$


$\mathrm{y}$ entonces tenemos que

$$
H_{0}^{T}=\frac{-\delta_{1} a}{2}\left(x_{1}^{2}+y_{1}^{2}\right)+\frac{-\delta_{2}}{2}\left(y_{2}^{2}+y_{3}^{2}\right)+a\left(x_{2} y_{3}-x_{3} y_{2}\right) .
$$

Por lo tanto,

$$
\left(\nabla H_{0}^{T}\right)^{T}=\left(-\delta_{1} a x_{1}, a y_{3},-a y_{2},-\delta_{1} a y_{1},-\delta_{2} y_{2}-a x_{3},-\delta_{2} y_{3}+a x_{2}\right)
$$

luego, para un polinomio homogéneo de orden $s>2, K^{s}$, tenemos

$$
\begin{gathered}
\left\{H_{0}^{T}, K^{s}\right\}=-\delta_{1} a\left(x_{1} \frac{\partial K^{s}}{\partial y_{1}}-y_{1} \frac{\partial K^{s}}{\partial x_{1}}\right)-a\left(x_{2} \frac{\partial K^{s}}{\partial x_{3}}-x_{3} \frac{\partial K^{s}}{\partial x_{2}}\right) \\
-a\left(y_{2} \frac{\partial K^{s}}{\partial y_{3}}-y_{3} \frac{\partial K^{s}}{\partial y_{2}}\right)+\delta_{2}\left(y_{2} \frac{\partial K^{s}}{\partial x_{2}}+y_{3} \frac{\partial K^{s}}{\partial x_{3}}\right)
\end{gathered}
$$

Sea $L$ el operador diferencial,

$$
\begin{gathered}
L=\delta_{1} a\left(x_{1} \frac{\partial}{\partial y_{1}}-y_{1} \frac{\partial}{\partial x_{1}}\right)+a\left[\left(x_{2} \frac{\partial}{\partial x_{3}}-x_{3} \frac{\partial}{\partial x_{2}}\right)+\left(y_{2} \frac{\partial}{\partial y_{3}}-y_{3} \frac{\partial}{\partial y_{2}}\right)\right] \\
-\delta_{2}\left(y_{2} \frac{\partial}{\partial x_{2}}+y_{3} \frac{\partial}{\partial x_{3}}\right) .
\end{gathered}
$$

Entonces la condición (1.4) es equivalente a que $L\left(K^{s}\right)=0, \quad s=3, \ldots$, para un polinomio homogéneo de grado $s, K^{s} \in \operatorname{Ker}\left(L_{A^{T}}^{s}\right)$.

Consideremos el cambio de coordenadas siguiente,

$$
\begin{aligned}
& \zeta_{1}=x_{1}+i y_{1}, \quad \zeta_{2}=x_{2}+i x_{3}, \quad \zeta_{3}=y_{2}+i y_{3} \\
& \bar{\zeta}_{1}=x_{1}-i y_{1}, \quad \bar{\zeta}_{2}=x_{2}-i x_{3}, \quad \bar{\zeta}_{3}=y_{2}-i y_{3} .
\end{aligned}
$$

Esta no es una transformación simpléctica, pero a través de ella mostraremos que en las coordenadas simplécticas $x_{j}, y_{j}, \operatorname{Ker}\left(L_{A^{T}}^{s}\right)$ tiene la forma especial enunciada en el Teorema.

En las coordenadas $\zeta_{j}, \bar{\zeta}_{j}$, tenemos

$$
\begin{aligned}
L\left(\zeta^{k} \bar{\zeta}^{l}\right)= & \zeta^{k} \bar{\zeta}^{l} i a\left\{\delta_{1}\left(k_{1}-l_{1}\right)+\left(k_{2}-l_{2}\right)+\left(k_{3}-l_{3}\right)\right\} \\
& -\delta_{2} \zeta^{k} \bar{\zeta}^{l}\left\{k_{2} \zeta_{2}^{-1} \zeta_{3}+l_{2} \bar{\zeta}_{2}^{-1} \bar{\zeta}_{3}\right\}
\end{aligned}
$$

y como

$$
K^{s}(\zeta, \bar{\zeta})=\sum_{|k|+|l|=s} h_{k l} \zeta^{k} \bar{\zeta}^{l}, \quad h_{k l} \in \mathbb{C}
$$

obtenemos que, para que un polinomio homogéneo $K^{s}$ esté en $\operatorname{Ker}\left(L_{A^{T}}\right)$, debe satisfacerse

$$
\begin{aligned}
L\left(K^{s}\right)= & \sum_{|k|+|l|=s} h_{k l} \zeta^{k} \bar{\zeta}^{l} i a\left\{\delta_{1}\left(k_{1}-l_{1}\right)+\left(k_{2}-l_{2}\right)+\left(k_{3}-l_{3}\right)\right\} \\
& -\delta_{2} \sum_{|k|+|l|=s} h_{k l} \zeta^{k} \bar{\zeta}^{l}\left\{k_{2} \zeta_{2}^{-1} \zeta_{3}+l_{2} \bar{\zeta}_{2}^{-1} \bar{\zeta}_{3}\right\} \\
& =0,
\end{aligned}
$$


es decir,

$$
\begin{aligned}
& \sum_{|k|+|l|=s} h_{k l} \zeta^{k} \bar{\zeta}^{l} i a\left\{\delta_{1}\left(k_{1}-l_{1}\right)+\left(k_{2}-l_{2}\right)+\left(k_{3}-l_{3}\right)\right\} \\
- & \delta_{2} \sum_{|k|+|l|=s} h_{k l}\left\{k_{2} \zeta_{2}^{k_{2}-1} \zeta_{3}^{k_{3}+1} \bar{\zeta}_{2}^{l_{2}} \bar{\zeta}_{3}^{l_{3}}+l_{2} \zeta_{2}^{k_{2}} \zeta_{3}^{k_{3}} \bar{\zeta}_{2}^{l_{2}-1} \bar{\zeta}_{3}^{l_{3}+1}\right\} \zeta_{1}^{k_{1}} \bar{\zeta}_{1}^{l_{1}} \\
= & \sum_{|k|+|l|=s}\left\{h_{k l} i a\left\langle k-l,\left(\delta_{1}, 1,1\right)\right\rangle-\delta_{2}\left[k_{2}+1 h_{\left(k_{1}, k_{2}+1, k_{3}-1, l_{1}, l_{2}, l_{3}\right)}\right.\right. \\
+ & \left.\left.\left(l_{2}+1\right) h_{\left(k_{1}, k_{2}, k_{3}, l_{1}, l_{2}+1, l_{3}-1\right)}\right]\right\} \zeta^{k} \bar{\zeta}^{l} \\
= & 0
\end{aligned}
$$

De aquí obtenemos el sistema de ecuaciones,

$$
\begin{aligned}
& h_{k l} i a\left\langle k-l,\left(\delta_{1}, 1,1\right)\right\rangle \\
& -\delta_{2}\left[\left(k_{2}+1\right) h_{\left(k_{1}, k_{2}+1, k_{3}-1, l_{1}, l_{2}, l_{3}\right)}+\left(l_{2}+1\right) h_{\left(k_{1}, k_{2}, k_{2}, l_{1}, l_{2}+1, l_{3}-1\right)}\right] \\
& =0
\end{aligned}
$$

pues los $\left\{\zeta^{k} \bar{\zeta}^{l}\right\}$ son linealmente independientes sobre $\mathbb{C}$. Equivalentemente, tenemos el sistema

$$
\begin{aligned}
& h_{k l} a\left\langle k-l,\left(\delta_{1}, 1,1\right)\right\rangle \\
& (3.6)=-i \delta_{2}\left[\left(k_{2}+1\right) h_{\left(k_{1}, k_{2}+1, k_{3}-1, l_{1}, l_{2}, l_{3}\right)}+\left(l_{2}+1\right) h_{\left(k_{1}, k_{2}, k_{3}, l_{1}, l_{2}+1, l_{3}-1\right)}\right] .
\end{aligned}
$$

Consideremos el subconjunto de $\mathbb{Z}^{3} \times \mathbb{Z}^{3}$ definido por:

$M_{\delta_{1}}=\left\{r=(k, l)=\left(k_{1}, k_{2}, k_{3}, l_{1}, l_{2}, l_{3}\right) \in \mathbb{Z}_{\geq 0}^{3} \times \mathbb{Z} \geq 0^{3} /\left\langle k-l,\left(\delta_{1}, 1,1\right)\right\rangle=0\right\}$. Si $(k, l) \notin M_{\delta_{1}},\left\langle k-l,\left(\delta_{1}, 1,1\right)\right\rangle \neq 0$ y de (??) tenemos los siguientes casos:

i) $k_{3}=0 \mathrm{y} / \mathrm{o} l_{3}=0$. En este caso $h_{k l}=0$, pues los términos de exponentes, $\left(k_{1}, k_{2}+1,-1, l_{1}, l_{2}, l_{3}\right)$ y $\left(k_{1}, k_{2}, k_{3}, l_{1}, l_{2}+1,-1\right)$ no están en $K^{s}(\zeta, \bar{\zeta})$ y, entonces, los coeficientes de tales términos son nulos.

ii) $k_{3} \neq 0$ y $l_{3} \neq 0$. Supongamos $l_{3}>0$ y $k_{3}=1$. Entonces,

$$
\begin{aligned}
& h_{\left(k_{1}, k_{2}, 1, l_{1}, l_{2}, l_{3}\right)} \\
& =\frac{\left(-i \delta_{2}\right)^{l_{3}}\left(l_{2}+l_{3}\right) !}{l_{2} !\left\langle k-l,\left(\delta_{1}, 1,1\right)\right\rangle^{l_{3}} a^{l_{3}}} h_{\left(k_{1}, k_{2}, 1, l_{1}, l_{2}+l_{3}, 0\right)} \\
& =0
\end{aligned}
$$

Supóngase que, para $l_{3}>0$ y $k_{3} \leq \mathrm{p}$, se tiene $h_{k l}=0$. Entonces

$$
\begin{aligned}
& h_{\left(k_{1}, k_{2}, p+1, l_{1}, l_{2}, l_{3}\right)} \\
& =\frac{-i \delta_{2}}{\left\langle k-l,\left(\delta_{1}, 1,1\right)\right\rangle a}\left[\left(k_{2}+1\right) h_{\left(k_{1}, k_{2}+1, p, l_{1}, l_{2}, l_{3}\right)}\right. \\
& \left.+\left(l_{2}+1\right) h_{\left(k_{1}, k_{2}, p+1, l_{1}, l_{2}+1, l_{3-1}\right)}\right] \\
& =\frac{\left(-i \delta_{2}\right)^{l_{3}}\left(l_{2}+l_{3}\right) !}{l_{2} !\left\langle k-l,\left(\delta_{1}, 1,1\right)\right\rangle^{l_{3}} a^{l_{3}}} h_{\left(k_{1}, k_{2}, p+1, l_{1}, l_{2}+l_{3}, 0\right)} \\
& =0
\end{aligned}
$$


pues, $h_{\left(k_{1}, k_{2}+1, p, l_{1}, l_{2}+j, l_{3}-j\right)}=0, \quad j=0,1,2, \ldots$, por hipótesis. Luego, por inducción, $h_{k l}=0$, para todo $(k, l)$ con $l_{3}>0, k_{3}>0$. Se sigue de aquí que $h_{k l}=0$, para todo $(k, l) \notin M_{\delta_{1}}$, independientemente de $\delta_{2}$.

Si $(k, l) \in M_{\delta_{1}}$, de (3.6) tenemos que,

$$
k_{2} h_{\left(k_{1}, k_{2}, k_{3}, l_{1}, l_{2}, l_{3}\right)}=-\left(l_{2}+1\right) h_{\left(k_{1}, k_{2}-1, k_{3}+1, l_{1}, l_{2}+1, l_{3}-1\right)},
$$

donde $\delta_{1} k_{1}+k_{2}+k_{3}=\delta_{1} l_{1}+l_{2}+l_{3}$, para todo $(k, l)$ con $|k|+|l|=s$. Por lo tanto, si $K^{s} \neq 0, s$ es un número par, independiente de $\delta_{1}$.

iii) Consideremos $k_{1}$ y $l_{1}$ fijos. Sea $k_{2} \leq l_{3}$ ( o equivalentemente $k_{3} \geq l_{2}$ ). Considerando el orden parcial en los $(k, l)$, definida por,

$$
(k, l) \prec\left(k^{\prime}, l^{\prime}\right) \text {, si y sólo si, } l_{2}<l_{2}^{\prime}
$$

obtenemos la siguiente sucesión ordenada de sub-índices $(k, l)$ relacionados por la igualdad (3.7),

$$
\begin{gathered}
\left(k_{1}, k_{2}+l_{2}-j, k_{3}-l_{2}+j, l_{1}, j, k_{2}+k_{3}-j\right):=(j), \\
j=0,1,2, \ldots, k_{2}+l_{2}
\end{gathered}
$$

y, por (3.7),

$$
h_{(0)}=(-1)^{j} \frac{j !\left(k_{2}+l_{2}-j\right) !}{\left(k_{2}+l_{2}\right) !} h_{(j)}, \quad j=1,2, \ldots, k_{2}+l_{2},
$$

es decir ,

$$
h_{(j)}=(-1)^{j}\left(\begin{array}{c}
k_{2}+l_{2} \\
j
\end{array}\right) h_{(0)}, \quad j=1, \ldots, k_{2}+l_{2} .
$$

Sumando todos los términos en $K^{s}$, correspondientes a los sub-í ndices de la sucesión (3.9) y considerando (3.10), con $Z=(\zeta, \bar{\zeta})$, obtenemos

$$
\begin{aligned}
& \sum_{j=0}^{k_{2}+l_{2}} h_{(j)} Z^{(j)} \\
& =\left[\sum_{j=0}^{k_{2}+l_{2}}(-1)^{j}\left(\begin{array}{c}
k_{2}+l_{2} \\
j
\end{array}\right) \zeta_{2}^{k_{2}+l_{2}-j} \bar{\zeta}_{2}^{j} \zeta_{3}^{j} \bar{\zeta}_{3}^{k_{2}+l_{2}-j}\right] \zeta_{1}^{k_{1}} \bar{\zeta}_{1}^{l_{1}} \zeta_{3}^{k_{3}-l_{2}} \bar{\zeta}_{3}^{k_{3}-l_{2}} h_{(0)} \\
& =\left(\zeta_{2} \bar{\zeta}_{3}-\bar{\zeta}_{2} \zeta_{3}\right)^{k_{2}+l_{2}}\left(\zeta_{1}^{k_{1}} \bar{\zeta}_{1}^{l_{1}}\right)\left(\zeta_{3} \bar{\zeta}_{3}\right)^{k_{3}-l_{2}} h_{(0)}
\end{aligned}
$$

De la misma forma, si $k_{2}>l_{3}$ ( o equivalentemente, $k_{3}<l_{2}$ ) y para $k_{1}$ y $l_{1}$ fijos, obtenemos una sucesión de sub-índices

$$
\left(k_{1}, k_{2}+k_{3}-j, j, l_{1}, l_{2}-k_{3}+j, l_{3}+k_{3}-j\right):=(j), j=0,1,2, \ldots, l_{3}+k_{3}
$$


ordenados por un orden parcial similar al dado en (3.8), y entonces, sumando los términos correspondientes de $K^{s}$, obtenemos

$$
\begin{aligned}
& \sum_{j=0}^{k_{3}+l_{3}} h_{(j)} Z^{(j)} \\
= & {\left[\begin{array}{c}
k_{3}+l_{3} \\
\sum_{j=0}(-1)^{j}\left(\begin{array}{c}
k_{3}+l_{3} \\
j
\end{array}\right) \zeta_{2}^{k_{3}+l_{3}-j} \bar{\zeta}_{2}^{j} \zeta_{3}^{j} \bar{\zeta}_{3}^{k_{3}+l_{3}-j}
\end{array}\right] \zeta_{1}^{k_{1}} \bar{\zeta}_{1}^{l_{1}} \zeta_{2}^{l_{2}-k_{3}} \bar{\zeta}_{2}^{l_{2}-k_{3}} h_{(0)} } \\
= & \left(\zeta_{2} \bar{\zeta}_{3}-\bar{\zeta}_{2} \zeta_{3}\right)^{k_{3}+l_{3}}\left(\zeta_{1}^{k_{1}} \bar{\zeta}_{1}^{l_{1}}\right)\left(\zeta_{2} \bar{\zeta}_{2}\right)^{l_{2}-k_{3}} h_{(0)} .
\end{aligned}
$$

Considerando que

$$
\begin{gathered}
\Gamma_{2}=\frac{1}{2}\left(y_{2}^{2}+y_{3}^{2}\right)=\frac{1}{2} \zeta_{3} \bar{\zeta}_{3}, \\
\Gamma_{3}=x_{3} y_{2}-x_{2} y_{3}=\frac{-i}{2}\left(\zeta_{2} \bar{\zeta}_{3}-\bar{\zeta}_{2} \zeta_{3}\right), \\
\Gamma_{4}=\frac{1}{2}\left(x_{2}^{2}+x_{3}^{2}\right)=\frac{1}{2} \zeta_{2} \bar{\zeta}_{2}
\end{gathered}
$$

y sustituyendo en (3.11) y (3.12), obtenemos que los términos de $K^{s}$ pueden ser agrupados de tal forma que las sumas sólo dependan de las cantidades $\Gamma_{2}, \Gamma_{3}, \Gamma_{4}$, con exponentes enteros no negativos, y de los productos $\zeta_{1}^{k_{1}} \bar{\zeta}_{1}^{l_{1}}$; como $s$ es par y los $\Gamma_{j}$ aportan exponentes pares al grado de $K^{s}$, necesariamente en los exponentes de $\zeta_{1}^{k_{1}} \bar{\zeta}_{1}^{l_{1}}$ debe tenerse que $k_{1}+l_{1}$ es par.

Por lo expuesto, si $K^{s} \neq 0$ y $K^{s} \in \operatorname{Ker}\left(L_{A^{T}}\right)$, entonces

$$
K^{s}(\zeta, \bar{\zeta})=f^{s}\left(\zeta_{1}, \bar{\zeta}_{1}, \Gamma_{2}, \Gamma_{3}, \Gamma_{4}\right),
$$

donde $f^{s}$ es un polinomio homogéneo de orden $(s / 2)$ en $\zeta_{1}, \bar{\zeta}_{1}, \Gamma_{2}, \Gamma_{3}$ y $\Gamma_{4}$, con coeficientes en $\mathbb{C}$.

Además, $\left\{H_{0}^{T}, K^{s}\right\}=0$ implica que

$$
\begin{aligned}
\left\{H_{0}^{T}, K^{s}\right\}= & \delta_{1} a\left[x_{1}\left(K_{\zeta_{1}}^{s} i+K_{\bar{\zeta}_{1}}^{s}(-i)\right)-y_{1}\left(K_{\zeta_{1}}^{s}+K_{\bar{\zeta}_{1}}^{s}\right)\right] \\
& -a\left[x_{2}\left(K_{\Gamma_{3}}^{s} y_{2}+K_{\Gamma_{4}}^{s} x_{3}\right)-x_{3}\left(-K_{\Gamma_{3}}^{s} y_{3}+K_{\Gamma_{4}}^{s} x_{2}\right)\right] \\
& -a\left[y_{2}\left(-K_{\Gamma_{3}}^{s} x_{2}+K_{\Gamma_{2}}^{s} y_{3}\right)-y_{3}\left(K_{\Gamma_{3}}^{s}+K_{\Gamma_{2}}^{s} y_{2}\right)\right] \\
& +\delta_{2}\left[y_{2}\left(-K_{\Gamma_{3}}^{s} y_{3}+K_{\Gamma_{4}}^{s} x_{2}\right)+y_{3}\left(K_{\Gamma_{3}}^{s} y_{2}+K_{\Gamma_{4}}^{s} x_{3}\right)\right] \\
= & i \delta_{1} a\left[K_{\zeta_{1}}^{s} \zeta_{1}-K_{\bar{\zeta}_{1}}^{s} \bar{\zeta}_{1}\right]+\delta_{2}\left[x_{2} y_{2}+x_{3} y_{3}\right] K_{\Gamma_{4}}^{s} \\
= & 0
\end{aligned}
$$

con $s>2$. De aquí obtenemos la igualdad de polinomios

$$
i \delta_{1} a\left[K_{\zeta_{1}}^{s} \zeta_{1}-K_{\bar{\zeta}_{1}}^{s} \bar{\zeta}_{1}\right]=-\delta_{2}\left[x_{2} y_{2}+x_{3} y_{3}\right] K_{\Gamma_{4}}^{s} .
$$


Como $K^{s}$ tiene la forma

$$
K^{s}=\sum_{k_{1}+l_{1}+2\left(n_{2}+n_{3}+n_{4}\right)=s} h_{\left(k_{1}, l_{1}, n_{2}, n_{3}, n_{4}\right)} \zeta_{1}^{k_{1}} \bar{\zeta}_{1}^{l_{1}} \Gamma_{2}^{n_{2}} \Gamma_{3}^{n_{3}} \Gamma_{4}^{n_{4}},
$$

(3.14) es equivalente a

$$
\begin{aligned}
& i \delta a \sum_{k_{1}+l_{1}+2\left(n_{2}+n_{3}+n_{4}\right)=s}\left(k_{1}-l_{1}\right) h_{\left(k_{1}, l_{1}, n_{2}, n_{3}, n_{4}\right)} \zeta_{1}^{k_{1}} \bar{\zeta}_{1}^{l_{1}} \Gamma_{2}^{n_{2}} \Gamma_{3}^{n_{3}} \Gamma_{4}^{n_{4}} \\
& =-\delta_{2} \sum_{k_{1}+l_{1}+2\left(n_{2}+n_{3}+n_{4}\right)=s} n_{4} h_{\left(k_{1}, l_{1}, n_{2}, n_{3}, n_{4}\right)} \zeta_{1}^{k_{1}} \bar{\zeta}_{1}^{l_{1}} \Gamma_{2}^{n_{2}} \Gamma_{3}^{n_{3}} \Gamma_{4}^{n_{4}-1}
\end{aligned}
$$

Comparando exponentes (en $x_{2}$ o $x_{3}$ ), necesariamente ambos miembros en (3.13) deben ser nulos. Entonces tenemos que

- $k_{1}=l_{1}$ o $h_{\left(k_{1}, l_{1}, n_{2}, n_{3}, n_{4}\right)}=0$, si consideramos el primer miembro, y

- $n_{4}=0$ o $h_{\left(k_{1}, l_{1}, n_{2}, n_{3}, n_{4}\right)}=0$, si consideramos el segundo, lo que implica que $K^{s}$ no depende de $\Gamma_{4}$.

De lo anterior tenemos que si

$$
\Gamma_{1}=\frac{1}{2} \zeta_{1} \bar{\zeta}_{1}=\frac{1}{2}\left(x_{1}^{2}+y_{1}^{2}\right)
$$

$K^{s} \in \operatorname{Ker}\left(L_{A^{T}}^{s}\right), K^{s} \neq 0$ con $s>2$, entonces $s$ es par y $K^{s}=f^{s}\left(\Gamma_{1}, \Gamma_{2}, \Gamma_{3}\right)$, donde $f^{s}$ es un polinomio homogéneo en $\Gamma_{1}, \Gamma_{2}, \Gamma_{3}$ de orden $\frac{s}{2}$ con coeficientes reales. Notemos que utilizando la transformacin inversa, es decir, de las coordenadas complejas a las reales originales, la expresión de $K^{s}$ no es alterada, por lo que está demostrada la parte (I) del Teorema.

II) La parte (II) es una consecuencia inmediata de la parte (I), pues todos los términos $H^{s}$ de grado $s+2$, con $s>0$, de $H^{*}$ están en $\operatorname{Ker}\left(L_{A^{T}}^{s+2}\right)$ y $H^{0}$ mantiene su forma normal original.

Luego, el Teorema está demostrado.

\section{References}

[1] Arnold V. I. : Mathematical Methods of Classical Machanics. SpringerVerlag New York, Inc., (1978). 
[2] Laub, A. J. and Meyer, K.: Canonical Forms for Symplectic and Hamiltonian Matrices. Celestial Mechanics, Vol. 9, pp. 233-238, (1974).

[3] Mansilla, J. E.: Sobre el núcleo del operador de Lie en el caso de tres grados de libertad y raí ces imaginarias puras con dos frecuencias iguales. Pre-print, (2001).

[4] Meyer, K. R. and Hall, G.R.: Introduction to Hamiltonian Dynamical System and the N-Body Problem. Springer-Verlag, New York, (1992).

[5] Sokol'skii, A. G.: On the stability of autonomous Hamiltonian system with two degrees of freedom in the case equal frequencies. P. M. M., Vol. 38, pp. 741-749, (1974).

\section{José Mansilla}

Departamento de Matemática y Física, Universidad de Magallanes

Casilla 113-D

Punta Arenas

Chile

e-mail : emansi@ona.fi.umag.cl 